\title{
POTENCIAL IMUNOMODULATÓRIO DA LECTINA DE INFLORESCENCIAS DE ALPINIA PURPURATA (ApuL) FRENTE A NEUTRÓFILOS HUMANOS
}

\author{
B. R. BARBOZA ${ }^{1}$, J. S. BRITO ${ }^{2}$, R. R. AZEVEDO ${ }^{3}$, M. C. A. ROQUE-BARREIRA ${ }^{3}$, T. H.
} NAPOLEÃO ${ }^{2}$, C. M. L. DE MELO ${ }^{4}$

${ }^{1}$ Centro Universitário Estácio do Recife, Bacharelado em Farmácia;

${ }^{2}$ Universidade Federal de Pernambuco, Centro de Biociências, Departamento de Bioquímica, Laboratório de Bioquímica de Proteínas;

${ }^{2}$ Universidade de São Paulo, Faculdade de Medicina de Ribeirão Preto, Departamento de Biologia Celular e Molecular e Bioagentes Patogênicos, Laboratório de Imunoquímica e Glicobiologia;

${ }^{3}$ Universidade Federal de Pernambuco, Centro de Biociências, Departamento de Antibióticos, Laboratório de Análises Imunológicas e Antitumorais

E-mail para contato: brunor.barbozza@gmail.com

RESUMO - As plantas são constituidas por compostos bioativos de interesse farmacológico para a medicina tradicional e moderna. Dentre estes compostos vegetais, estão as lectinas, que são proteinas com capacidade de reconhecimento de carboidratos. As lectinas ligam-se as estruturas glicanas de maneira especifica, porém reversível, e estão envolvidas em mecanismos de sinalização celular, apresentam potencial antimicrobiano, antitumoral e capacidade imunomodulatória. A lectina ApuL, alvo de estudo desse trabalho, é purificada da inflorescência de Alpinia purpurata. O objetivo deste estudo foi avaliar a potencial capacidade imunomodulatória de ApuL frente a neutrófilos humanos. Após a coleta do sangue de doadores voluntários, os neutrófilos foram isolados e tratados com ApuL nas concentrações 1,25 e 2,5 $\mathrm{gg} / \mathrm{mL}$ por 24 horas. Os níveis das citocinas $I L-1 \beta, I L-8, I L-10, I L-12 p 40, T N F-\alpha$ e TGF- $\beta 1$ foram quantificados nos sobrenadantes de cultura utilizando testes imunoenzimáticos (ELISA). Nossos resultados sugerem que os neutrófilos tratados com ApuL sofreram estimulação significativa podendo contribuir com a modulação da resposta adaptativa, especialmente em células $T \mathrm{CD}^{+}$. Em experimentos futuros pretendemos investigar a capacidade de resposta microbicida de neutrófilos estimulados por ApuL.

PALAVRAS-CHAVE: ApuL; imunomodulação; lectina; neutrófilos; Th1/Th17.

ABSTRACT - Plants are constituted by bioactive compounds of pharmacological interesting to traditional and recent medicine. Lectins, proteins which recognize carbohydrates, are one of these compounds. These proteins binding specifically to glycan structures and are involved in cellular signalling mechanisms, showing immunomodulatory profile and antimicrobial and antitumor potential. ApuL lectin, goal of this study, is purified from the Alpinia purpurata inflorescence, and here, 
we evaluated the potential immunological ability of ApuL in human neutrophils. After blood collection of volunteers donors, neutrophils were isolated and treated in cultures with 1.25 and $2.5 \mu \mathrm{g} / \mathrm{mL}$ concentrations for 24 hours of assay. Cytokines $I L-1 \beta, I L-8, I L-10, I L-12 p 40, T N F-\alpha$ e TGF- $\beta 1$ levels were measured through culture supernatant using enzyme-linked immunosorbent assay (ELISA). Our results showed which neutrophils treated with ApuL presented significant stimulation and this behavior could have contributed with adaptive immune response modulation, especially in $T C D 4+$ cells. In future assays we will investigate the microbicide response ability of neutrophil stimulated with ApuL.

KEYWORDS: ApuL; immunomodulation; lectin; neutrophils; Th1 / Th17

\section{INTRODUÇÃO}

Atualmente, muitos dos fármacos comercialmente disponíveis, foram gerados a partir de produtos naturais e/ou derivados de plantas (Lahlou, 2013). De fato, as plantas têm sido utilizadas, desde a antiguidade, com finalidade medicinal, por constituírem umas das principais fontes de biomoléculas com potencial terapêutico (Atanasov et al., 2015). Dentre esses constituintes, estão as lectinas, que são proteínas com especificidade de ligação às estruturas glicanas nas superfícies celulares (Gorashakar e Ghosh, 2016). Nas plantas as lectinas estão distribuídas em diversos tecidos como raízes, folhas, flores, cerne, rizomas, tubérculos, vagens, entrecascas e frutos (Ingale et al., 2013) e estão envolvidas principalmente com os mecanismos defensivos das plantas (Sá et al., 2009).

Devido às suas aplicabilidades como valiosas ferramentas em diversas áreas como biologia molecular, imunologia e farmacologia, as lectinas têm sido alvo de relevantes pesquisas como a investigação e avaliação do potencial antibacteriano e antibiofilme (Moura et al., 2017) e antiviral e antitumoral (Xu et al., 2015). Além dessas propriedades, diversos estudos demonstram a capacidade imunomodulatória dessas proteínas, como, efeito mitogênico (Melo et al., 2011), produção de citocinas, liberação de óxido nítrico, ativação e proliferação celular (Patriota et al., 2017; Santana et al., 2017; Melo et al., 2010).

O sistema imunológico, constituído por células e moléculas ativas encontradas por todo o organismo, é caracterizado biologicamente pela capacidade de reconhecimento especifico à determinadas estruturas moleculares e/ou antígenos, promovendo resposta efetora. Dentre as células imunológicas da linhagem inata, os neutrófilos, destacam-se como os fagócitos circulantes mais abundantes (Oliveira et al., 2016). Estas células desenvolvem seu mecanismo efetor por três principais estratégias: a fagocitose, degranulação e formação de armadilhas extracelulares de neutrófilos (Kolaczkowska e Kubes, 2013). Além disso, estudos recentes atribuem aos neutrófilos importante papel na biologia do câncer (Coffelt et al., 2016) e atuação como células apresentadoras de antígenos, contribuindo assim com a modulação da resposta imunológica por linfócitos T (Takashima e Yao, 2015).

Alpinia purpurata (Vieill.) K. Schum é uma planta da família Zingiberaceae, nativa das ilhas do Pacífico e comumente encontrada em jardins, devido ao seu perfil ornamental, é vastamente cultivada no Brasil e na Índia (Santana et al., 2017; Raj et al., 2012). Estudos relatam diversas propriedades biológicas para esta espécie, tais como as atividades antimicrobianas (Kochuthressia et al., 2010), antioxidantes (Subramanian e Suja, 2011) e propriedade antitumoral (Oirere et al., 2016). A lectina ApuL, alvo de 
estudo deste trabalho é purificada de inflorescências da planta Alpinia purpurata, cujos estudos iniciais, recentemente publicados, demonstraram a sua capacidade de ativação e imunomodulação em PBMC humano. O objetivo deste estudo foi avaliar o potencial imunomodulador de ApuL sobre neutrófilos humanos, no que diz respeito a investigação da produção de citocinas e avaliação do perfil de resposta imunológica induzido pela lectina.

\section{MATERIAIS E MÉTODOS}

As inflorescências de $A$. purpurata foram coletadas no campus da Universidade Federal de Pernambuco - UFPE, autorizados pelo Instituto Chico Mendes para Conservação da Biodiversidade - ICMBio do Ministério do Meio Ambiente, com autorização $n^{\circ}$ 36301. A identificação botânica foi realizada pelo Herbário UFP Geraldo Muniz, CB - UFPE. Uma exsicata encontra-se depositada sob. $n^{\circ}$ 53.376. A lectina ApuL foi purificada conforme metodologia descrita por Santana et al. (2017). Após a coleta de sangue de doadores voluntários, os neutrófilos foram isolados conforme metodologia descrita por Ricci-Azevedo et al., (2016) e tratados com ApuL nas concentrações 1,25 e $2,5 \mu \mathrm{g} / \mathrm{mL}$. Os níveis das citocinas IL-1 $\beta$, IL-8, IL-10, IL-12 p40, TNF- $\alpha$ (BDBiosciences, EUA) e TGF- $\beta 1$ (D\&D Systems, EUA), foram quantificados nos sobrenadantes, por ELISA, segundo recomendações do fabricante. Utilizando o software GraphPad Prism (versão 6.0) realizamos as análises estatísticas, pelo teste de $t$ Student, One way ANOVA seguido pelo pós-teste de Bonferroni, e Two away ANOVA seguido do pós-teste de Bonferroni.

\section{RESULTADOS E DISCUSSÃO}

As citocinas desempenham importante papel na integralização das redes de sinalização entre várias células, tornando-as assim, essenciais para a regulação e desenvolvimento de processos imunes inatos e adaptativos (Feldmann, 2008; Silva et al., 2012). Em nossos estudos iniciais, recentemente publicados, ApuL foi capaz de induzir em PBMC a liberação de citocinas referentes a um perfil Th1 e Th17, sugerindo um fenótipo perfil de resposta Th1/Th17 (Santana et al., 2017), conforme resumo esquemático descrito na figura 1.

Figura 1 - Mecanismo molecular para a geração de fenótipos aditivos das células Th1 e Th17 demonstrado pela lectina ApuL em PBMC humano (Korn et al., 2009; Boniface et al., 2010; Lexberg et al., 2010; Cosmi et al., 2014; Tabarkiewicz et al., 2015; Santana et al., 2017).

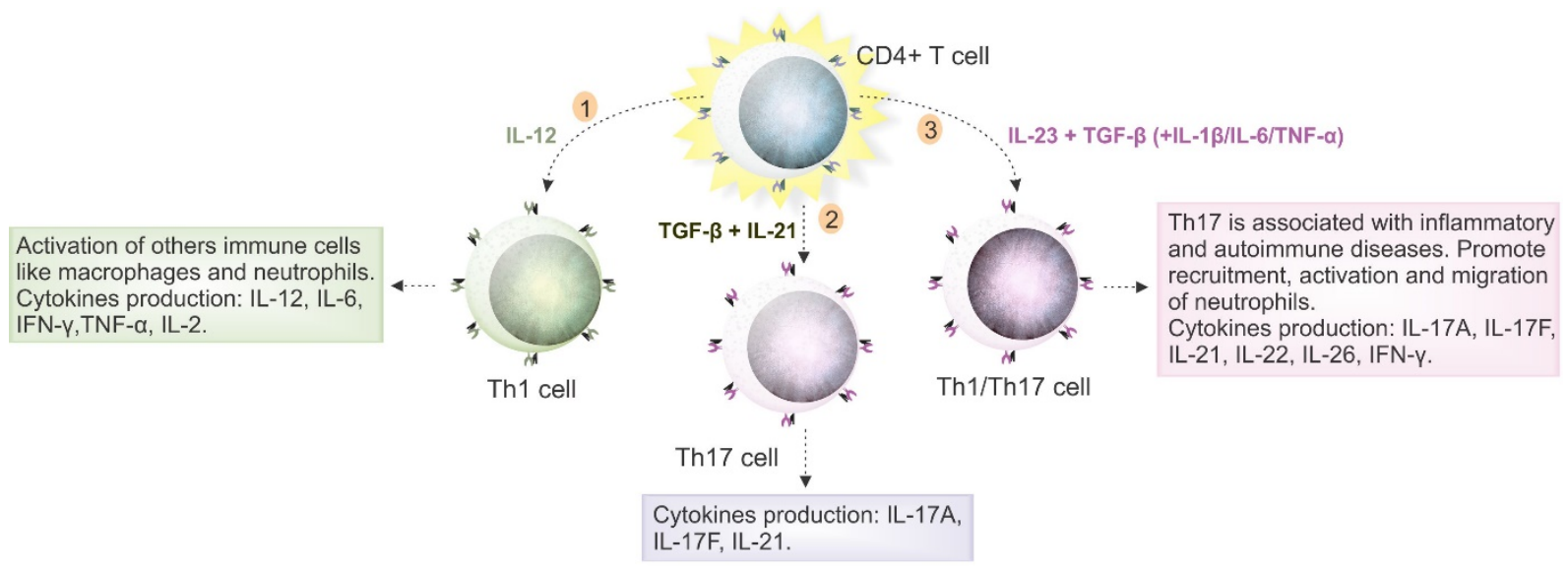


As lectinas atuam sobre a modulação da resposta imunológica mediada por neutrófilos, através da interação direta com a célula (induzindo a migração destas células para o sítio inflamatório, por exemplo). Além disso, as lectinas podem induzir uma resposta inflamatória aguda, caracterizada pelo elevado influxo de neutrófilos que migram para o sítio inflamatório, mediados por vários estímulos como microrganismos oportunistas, fatores quimiotáticos diversos e citocinas (Silva et al., 2012). Nossos resultados demonstraram que ApuL induziu, em neutrófilos, a liberação de citocinas pertencentes aos dois perfis de respostas imunológicas, ou seja, Th1 (IL-1 $\beta$, IL-12p40 e TNF- $\alpha$ ) e Th2 (IL-10 e TGF- $\beta$ ). Entretanto, houve prevalência da resposta Th1 (Figura 2A-E). Os resultados obtidos pela produção de citocinas corroboram com os estudos iniciais, tendo em vista que, estímulos por IL-23 associada à TGF- $\beta$, induzem a célula $\mathrm{T}$ $\mathrm{CD}^{+}$a maturar para a geração de fenótipos aditivos Th1/Th17. Além disso, este mesmo fenótipo pode ser induzido por estimulação conjunta com outras citocinas como IL-1 $\beta$, IL-6 e TNF- $\alpha$ (conforme observado na Figura 1) o que de fato ocorreu se associarmos os dois estudos imunológicos realizados com esta lectina. Nesse caso, sugerimos que neutrófilos tratados com ApuL, contribuem para a modulação da resposta imunológica adaptativa, mantendo a indução da geração de fenótipos aditivos Th1/Th17 por células $T$ $\mathrm{CD}^{+}$como descrito por Santana et al., (2017).

Citocinas pertencentes aos dois perfis (Th1 e Th17) possuem importante papel no direcionamento da resposta imunológica, especialmente, na proteção e eliminação contra patógenos. Estudos demonstram que esta associação (Th1/Th17) torna-se essencial na proteção contra infecção por M. tuberculosis, sem promover dano tecidual excessivo nos pulmões de indivíduos (Silva et al., 2015; Santarlasci et al., 2013). Haabeth e colaboradores (2011) demonstraram que a resposta imunológica promovida por células Th1 específicas em macrófagos, in vivo, foi capaz de erradicar linfoma de células B.

Figura 2. Perfil de produção de citocinas em neutrófilos humanos promovido pela ApuL. (A) ApuL foi capaz de produzir altas concentrações de IL-1 $\beta$ nas duas concentrações analisadas. O mesmo ocorreu para as citocinas IL12p40 (B) e TNF- $\alpha(\mathbf{C})$. A citocina IL10 foi produzida de forma significativa apenas na concentração de $2,5 \mu \mathrm{g} / \mathrm{mL}$ (D) e a citocina TGF- $\beta$ foi produzida em altos valores em $1,25 \mu \mathrm{g} / \mathrm{mL}(\mathbf{E})$.

A

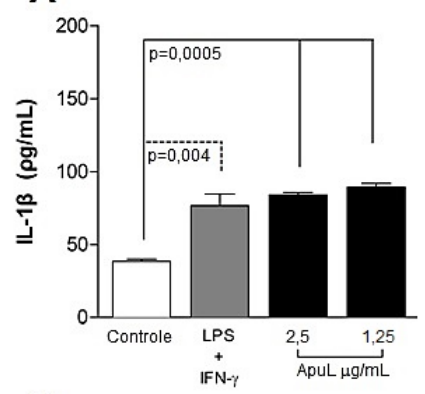

D

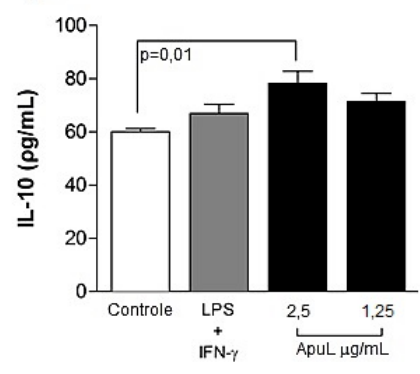

B

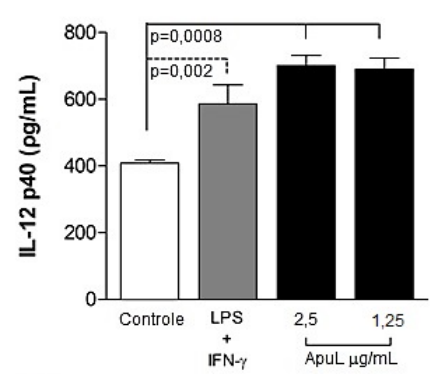

E

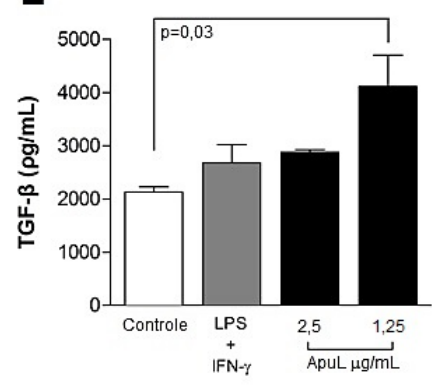

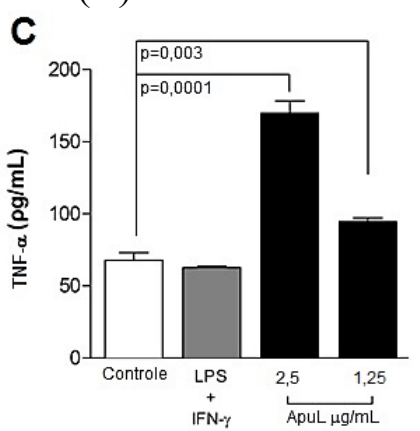


A citocina IL-10 (produzida em altos níveis sob influência de ApuL) pode ser produzida por células pertencentes aos perfis de respostas Th1, Th2 e/ou Th17 e têm importante papel influenciando na apresentação de antígenos, diferenciação de células $\mathrm{T}$ e produção de citocinas (Santana et al., 2017). Além disso, a IL-10 influencia a intensidade da inflamação modulando o processo inflamatório, na regulação de uma resposta controlada (Patriota et al., 2017; Silva et al., 2015).

\section{CONCLUSÃO}

Os resultados deste estudo, indicam que ApuL atua sobre neutrófilos humanos modulando o perfil de resposta imunológica, através da produção de citocinas. Além disso, sugerem futuros estudos na investigação do potencial biológico de ApuL (como um protótipo fármaco) candidato ao tratamento de infecções, bem como adjuvante nas terapias medicamentosas comercialmente disponíveis.

\section{REFERÊNCIAS}

ATANASOV, A. G.; WALTENBERGER, B.; PFERSCHY-WENZIG, E.-M.; et al. (2015). Discovery and resupply of pharmacologically active plantderived natural products: A review. Biotechnology Advances, 33(8), 1582-1614.

BONIFACE, K.; BLUMENSCHEIN, W.M.; BROVONT-PORTH, K.; MCGEACHY, M.J.; BASHAM, B.; DESAI, B.; PIERCE, R. et al. Human Th17 cells comprise heterogeneous subsets including IFN-gamma-producing cells with distinct properties from the Th1 lineage. J. Immunol. 2010. 185: 679-687.

COFFELT, S.B.; WELLENSTEIN, M.D.; DE VISSER, K.E. Neutrophils in cancer: neutral no more. Nat Rev Cancer. 2016 Jul;16(7):431-46.

COSMI, L.; MAGGI, L.; SANTARLASCI, V.; LIOTTA, F.; ANNUZIATO, F. T helper cells plasticity in inflammation. Cytometry A. 2014 Jan;85(1):36-42

OLIVEIRA, S; ROSOWSKI, E. E.; HUTTENLOCHER, A. Neutrophil migration in infection and wound repair: going forward in reverse. Nat Rev Immunol. 2016 May 27;16(6):378-91.

SANTANA , J.S.; FERREIRA, G.R.S.; KLIMCZAK, E.; GRYSHUK, L.; DE LIMA SANTOS, N.D; DE SIQUEIRA PATRIOTA, L.L.; MOREIRA, L.R.; SOARES, A.K.A.; BARBOZA, B.R.; PAIVA, P.M.G.; DO AMARAL FERRAZ NAVARRO, D.M.; DE LORENA, V.M.B; DE MELO, C.M.L; CORIOLANO, M.C.; NAPOLEÃO, T.H. Lectin from inflorescences of ornamental crop Alpinia purpurata acts on immune cells to promote Th1 and Th17 responses, nitric oxide release, and lymphocyte activation. Biomed Phamacother 2017 Oct;94:865-872.

FELDMANN, M. Many cytokines are very useful therapeutic targets in disease. J Clin Invest (2008) 118:3533-6.

GORAKSHAKAR, A.C.; GHOSH, A. Use of lectins in immunohematology. Asian $\boldsymbol{J}$ 
Transfus Sci. 2016 Jan-Jun; 10(1): 12-21.

HAABETH O.A.; LORVIK, H.B.; HAMMAMRSTRÖM, C.; DONALDSON, I.M.; HARALDSON, I.M et al. Inflammation driven by tumour-specific Th1 cells protects against B-cell câncer. Nat Commun 2011;2:240.

INGALE, A.G.; HIVRALE, A.U. Plant as a plenteous reserve of lectin. Plant Signaling \& Behavior 8:12, e26595; December 2013; (C) 2013 Landes Bioscience.

KOCHUTHRESSIA, KP; BRITTO, SJ; RAJ, JM; JASEENTHA, MO et al. (2010). Efficient regeneration of Alpinia purpurata (Vieill.) K. Schum. plantlets from rhizome bud explants. Intl. Res. J. of Plant Sci., 1(2): 043-047

KOLACZKOWSKA, E.; KUBES, P. Neutrophil recruitment and function in health and inflammation. Nat. Rev. Immunol. 13, 159-175 (2013).

KORN, T.; BETTELLI,E.; OUKKA, M.; KUCHROO, V.K. IL-17 and Th17 Cells. Annu. Rev. Immunol. 2009. 27:485-517.

LAHLOU, M (2013). The Success of Natural Products in Drug Discovery. Pharmacology \& Pharmacy. 4, 17-31.

LEXBERG, M.H.; TAUBNER, A.; ALBRECHT, I.; LEPENIES, I.; RICHTER, A. et al. IFN-c and IL-12 synergize to convert in vivo generated Th17 into Th1/Th17 cells. Eur. J. Immunol. 2010. 40: 3017-3027.

MELO, C.M.L.; CASTRO, M.C.A.B.; OLIVEIRA, A.P.; GOMES, F.O.S. et al. Immunomodulatory response of Cramoll 1,4 lectin on experimental lymphocytes, Phytother. Res. 24 (2010) 1631-1636.

MELO C.M.; MELO, H.; CORREIA, MT., COELHO, L.C. et al., Mitogenic response and cytokine production induced by cramoll 1,4 lectin in splenocytes of inoculated mice. Scand J Immunol (2011) Feb;73(2):112-21.

MOURA, M.C.; TRENTIN, D.S.; NAPOLEÃO, T.H.; PRIMON-BARROS, M.; XAVIER, A.S. et al. Multi-effect of the water-soluble Moringa oleifera lectin against Serratia marcescens and Bacillus sp.: antibacterial, antibiofilm and anti-adhesive properties. J Appl Microbiol. 2017 Oct;123(4):861-874.

OIRERE, EK; ANUSOORIYA, P; MALARVIZHI, D; RAJ, CA; GOPALAKRISHNAN, VK (2016). Antioxidant, cytotoxic and apoptotic activities of crude extract of Alpinia purpurata on Cervical Cancer Cell Line. Int. J. Pharm. Sci. Rev. Res., 36(2), Jan-Fev. No. 06, 28-34.

PATRIOTA, L.L.S.; PROCÓPIO, T.F.; BRITO, J.S.; SEBAG, V.; OLIVEIRA, A.P.S.; SOARES, A.K.A.; MOREIRA, L.R.; LIMA, T.A.; SOARES, T.; SILVA, T.D.; PAIVA, P.M.G.; LORENA, V.M.B.; MELO, C.M.L.; ALBUQUERQUE, L.P.; NAPOLEÃO, T.H. Microgramma vacciniifolia (Polypodiaceae) fronds contain a multifunctional lectin with immunomodulatory properties on human cells, Int. J. Biol. Macromol. 103 (2017) $36-46$.

RAJ, CA; RAGAVENDRAN, P; SOPHIA, D; RATHI, MA; GOPALAKRISHNAN, VK 
(2012). Evaluation of in vitro antioxidante and anticancer activity of Alpinia purpurata.

Chinese Journal of Natural Medicines. (10)4: 0263-0268.

RICCI-AZEVEDO, R.; OLIVEIRA, A.F.; CONRADO, M.C.; CARVALHO, F.C.; ROQUE-BARREIRA, M.C. Neutrophils Contribute to the Protection Conferred by ArtinM against Intracellular Pathogens: A Study on Leishmania major. PLoS Ngegl Trop Dis. 2016 Apr 8;10(4):e0004609. doi: 10.1371/journal.pntd.0004609. eCollection 2016 Apr.

SUBRAMANIAN, V; SUJA, S (2011). Evaluation of antioxidant activity of Alpinia purpurata rhizome (Vieill). Int. J. Pharm. Sci., 2(4), 601-607.

SÁ, R.A.; SANTOS, N.D.; DA SILVA, C.S.; NAPOLEÃO, T.H. et al. Larvicidal activity of lectins from Myracrodruon urundeuva on Aedes aegypti. Comp Biochem Physiol C Toxicol Pharmacol 2009 Apr;149(3):300-6. doi: 10.1016/j.cbpc.2008.08.004. Epub 2008 Aug 15.

SANTARLASCI ,V; COSMI,L.; MAGGI,L,. LIOTTA, F.; ANNUNZIATO, F. IL-1 and T helper immune responses. Frontiers imunnology. July 2013 | V.4 | Article 182.

SILVA, M.V.S.; TIBURCIO, M.G.S.; MACHADO, J.R.; SILVA, D.A.A. et al. Complexity and Controversies over the Cytokine Profiles of $\mathrm{T}$ Helper Cell Subpopulations in Tuberculosis. J Immunol Res. Volume 2015, Article ID 639107, 13 pages.

SILVA, G.; CARVALHO, F. C.; ROQUE-BARREIRA, M. C. (2012). Neutrophil activation induced by plant lectins: modulation of inflammatory processes. Inflamm. Allergy Drug Targets 11, 433-441

TAKASHIMA, A.; YAHO, Y. Neutrophil plasticity: acquisition of phenotype and functionality of antigen-presenting cell. J Leukoc Biol. 2015 Oct;98(4):489-96. doi: 10.1189/jlb.1MR1014-502R. Epub 2015 Jan 28.

TABARKIEWICZ, J.; POGODA, K.; KARCZMARCZYK, A.; PORAROWSKI, P. et al. The Role of IL-17 and Th17 Lymphocytes in Autoimmune Diseases. Archivum Immun et Ther Experimentalis. December 2015, Volume 63, Issue 6, pp 435-449

TECCHIO, C; MICHELETTI, A.; CASSATELLA, M.A. Neutrophil-Derived Cytokines: Facts Beyond Expression. Front Immunol. 2014; 5 : 508. Published online 2014 Oct 21

TERRA, R.; SILVA, S.A.G.; PINTO, V.S.; DUTRA, P.M.L. Efeito do exercício no sistema imune: resposta, adaptação e sinalização celular. Rev Bras Med Esporte vol.18 no.3 São Paulo May/June 2012.

XU, X.C.; ZHANG, Z.W.; CHEN. Y.E; YUAN, M.; YUAN, S.; BAO, J.K. Antiviral and antitumor activities of the lectin extracted from Aspidistra elatior. $Z$ Naturforsch C, 2015;70 (1-2):7-13. 


\section{AGRADECIMENTOS}

Os autores expressam sua gratidão ao laboratório de Imunoquímica e Glicobiologia do Departamento de Biologia Celular e Molecular e Bioagentes Patogênicos da Faculdade de Medicina de Ribeirão Preto-FMRP/USP. Ao Laboratório de Bioquímica de Proteínas do Departamento de Bioquímica e ao Laboratório de Análises Imunológicas e Antitumorais, do Departamento de Antibióticos, sendo ambos da Universidade Federal de Pernambuco. 